\title{
Boundary element computation of fresh water groundwater lenses
}

\author{
D. S. Holloway* $\quad$ L. K. Forbes ${ }^{\dagger}$
}

(Received 23 November 2005; revised 3 October 2006)

\begin{abstract}
Groundwater is often used as a source of potable water on tropical islands. Boundary integral techniques are used here to determine the shape of the fresh water lens floating over intruded salty groundwater beneath a two dimensional tropical island. These computations may be used to determine optimum well location and maximum pumping rates. This paper describes the application of boundary integrals to groundwater flow and discretisation of the integral for numerical evaluation. Solutions to some numerical difficulties encountered in this problem are presented. These include a pseudo-unsteady method of satisfying the highly nonlinear boundary conditions that define the salt-fresh water interface beneath and phreatic surface above the lens, and reduced element requirements by the use of a suitable Green's
\end{abstract}

*School of Engineering, University of Tasmania, Hobart, Australia. mailto:Damien. Holloway@utas . edu.au

$\dagger^{\dagger}$ School of Mathematics and Physics, University of Tasmania, Hobart, Australia.

See http://anziamj.austms.org.au/V47EMAC2005/Holloway for this article, (C) Austral. Mathematical Soc. 2006. Published December 15, 2006. ISSN 1446-8735 
function to represent the outflow surfaces at the sides of an island with sloping sides, obtained by conformal mapping. Finally, some results are presented and other applications are briefly discussed.

\section{Contents}

1 Introduction and mathematical description

2 Solution by boundary integral method: no sink

3 Inclusion of a pumping well within the lens

4 Complex representation

5 Green's function and its integrals

6 Some results

7 Conclusions

C459

References

C461

\section{Introduction and mathematical description}

Precipitation produces fresh groundwater, which in the absence of significant mixing may form a layer over denser salty groundwater. In a restricted domain, such as a tropical island, the fresh water layer may then form a lens whose shape is dependent on the groundwater flow, and vice versa. Knowledge of both the flow pattern and the lens shape is essential for quantifying the effect of extraction; for example for water supply. 


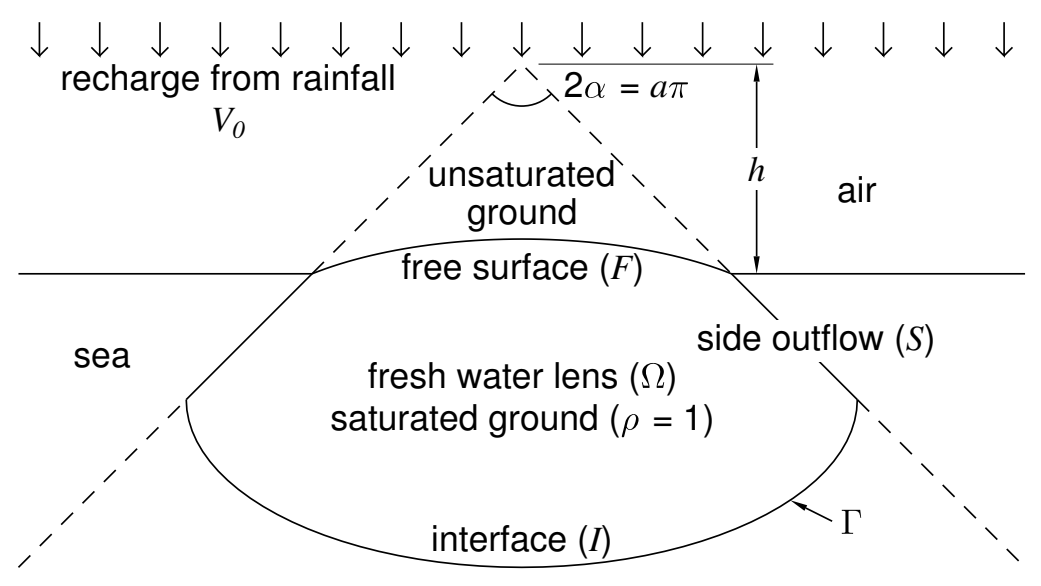

salt water saturated ground $(\rho=\gamma)$

FiguRE 1: Idealised fresh-water lens

An idealised representation of the subject of this paper is depicted in Figure 1, in which a steady-state groundwater flow within a two-dimensional island results in a lens of fresh water, recharged by effective precipitation (excluding evaporation and runoff), floating above a region of static salt water that has intruded from the surrounding sea. Fresh water is extracted by a pump located within the lens (not shown), and the pump location may be optimised for maximum extraction rate. In the idealisation the island's sides are assumed to be of constant slope (at least over the outflow area), and the ground is assumed to be hydraulically homogeneous and isotropic. The length unit for normalisation is the island half-width at sea level.

Within the ground there are three distinct regions: the underlying saltwater layer, which is static at steady state; the fresh-water lens $\Omega$, which is saturated; and the unsaturated zone above, in which there is no pore pressure. Separating these regions is a free surface (water table) $\mathcal{F}$ above $\Omega$ and a saltfresh water interface $\mathcal{I}$ below, the locations of which are to be determined as the primary task to be undertaken. In addition, $\mathcal{S}$ represents the two 
sides of the island between $\mathcal{F}$ and $\mathcal{I}$. Region $\Omega$ has inflow on $\mathcal{F}$ balanced by outflow at $\mathcal{S}$ (and extraction pump $W$ if present) and is bounded by contour $\Gamma=\mathcal{F} \cup \mathcal{S} \cup \mathcal{I}$.

A simplified version of the problem with vertical shores $\mathcal{S}$ and fixed horizontal upper surface $\mathcal{F}$ is solved in [2]. The present work extends this to sloping sides and unprescribed free surface.

Under the assumption of Darcy flow in a homogeneous isotropic soil, the groundwater flow is described by Laplace's equation,

$$
\nabla^{2} \Phi=0 \quad \text { in } \Omega,
$$

where after normalisation $\Phi$ represents the static head $y+p / \rho g$, and $-\nabla \Phi$ the effective or macroscopic flow velocity.

The locations of $\mathcal{I}$ and $\mathcal{F}$ are unknown, and hence two boundary conditions are required, whereas $\mathcal{S}$ is fixed and only requires one boundary condition. We impose the dynamic conditions that pressure vanishes on $\mathcal{F}$ and is governed by hydrostatics within the salt water on $\mathcal{I}$ and $\mathcal{S}$, leading to

$$
\Phi= \begin{cases}(1-\gamma) y, & \text { on } \mathcal{I} \cup \mathcal{S} \\ y, & \text { on } \mathcal{F} .\end{cases}
$$

Kinematic conditions of no normal flow and of normal flow compatible with the recharge are imposed on $\mathcal{I}$ and $\mathcal{F}$ respectively,

$$
-\nabla \Phi \cdot \mathbf{n}= \begin{cases}0, & \text { on } \mathcal{I} \\ V_{0}(-\mathbf{j}) \cdot \mathbf{n}, & \text { on } \mathcal{F} .\end{cases}
$$

Here $\gamma$ is the ratio of salt to fresh water density, $V_{0}$ is the recharge velocity normalised by the soil permeability, $\mathbf{j}$ is the unit vector pointing vertically, and $\mathbf{n}$ is the unit normal vector directed outwards from $\Omega$ on contour $\Gamma$. 


\section{Solution by boundary integral method: no sink}

Suppose $\Phi$ and $G$ are any two functions defined in the flow domain $\Omega$ such that $\nabla^{2} \Phi=0$ and $\nabla^{2} G=0$ except possibly at one or more points on $\Gamma$. For an arbitrary fixed point $Q$ on $\Gamma$, Green's second identity is

$$
\oint_{\Gamma}\left((\Phi(P)-\Phi(Q)) \frac{\partial G(P, Q)}{\partial n_{P}}-G(P, Q) \frac{\partial \Phi(P)}{\partial n_{P}}\right) d s_{P}=0 .
$$

Note from (3) that $\nabla \Phi \cdot \mathbf{n}$ is unspecified on $\mathcal{S}$ but can be eliminated from (4) by choosing any function $G$ harmonic in $\Omega$ such that

$$
G=0 \quad \text { on } \mathcal{S},
$$

(a suitable form for $G$ will be described below). Hence, defining $V_{n}$ as the sought value of $-\partial \Phi / \partial n$ given in (3), we seek $y$ on $\mathcal{I} \cup \mathcal{F}$ satisfying

$$
0=\gamma \int_{\mathcal{S} \cup \mathcal{I}} y \frac{\partial G}{\partial n} d s-\oint_{\Gamma} y \frac{\partial G}{\partial n} d s+y_{Q}(1-\gamma) \oint_{\Gamma} \frac{\partial G}{\partial n} d s+\int_{\mathcal{F}} V_{n} G d s
$$

for $Q \in \mathcal{I}$, or

$$
0=\gamma \int_{\mathcal{S} \cup \mathcal{I}} y \frac{\partial G}{\partial n} d s-\oint_{\Gamma} y \frac{\partial G}{\partial n} d s+y_{Q} \oint_{\Gamma} \frac{\partial G}{\partial n} d s+\int_{\mathcal{F}} V_{n} G d s
$$

for $Q \in \mathcal{F}$. These form a highly nonlinear set of equations in which the unknown $y$ appears implicitly in $\Gamma$ as well as explicitly.

The solution approach is to assume a trial location for the boundaries $\mathcal{I}$ and $\mathcal{F}$ and update them using the discrepancy between the quasi-unsteady boundary fluid normal velocity $V=-\nabla \Phi \cdot \mathbf{n}$, computed from

$$
\int_{\mathcal{F} \cup \mathcal{I}} V G d s=-\oint_{\Gamma} \Phi \frac{\partial G}{\partial n} d s+\Phi_{Q} \oint_{\Gamma} \frac{\partial G}{\partial n} d s
$$


on a trial boundary $\Gamma$ on which $\Phi$ given by (2), and the sought value $V_{n}$ given in (3). ${ }^{1} \quad$ Boundaries $\mathcal{I}$ and $\mathcal{F}$ are shifted by $\left(V-V_{n}\right) \Delta t$ for some suitable notional time step size $\Delta t$, see (17) and (18), until $V=V_{n}$, at which point (8) reduces to (6) or (7) and $\Gamma$ is the true boundary. A suitable $\Delta t$ is optimally chosen to be the largest $\Delta t$ for which stability and convergence are maintained - this is largely determined by numerical experimentation, but as a guiding principle will be generally in proportion to the size of the smallest element length.

Since $\Gamma$ may be of arbitrary shape we seek a numerical approximation to (8), obtained by representing $\Gamma$ by a set of ordered points $P_{i}(i=1, \ldots, N)$ that divide $\Gamma$ into straight line segments. The set of $P_{i}$ will be assumed to include the points $\mathcal{I} \cap \mathcal{S}$ and $\mathcal{F} \cap \mathcal{S}$, but need not contain other points on $\mathcal{S}$. Now define weight functions $w_{i}(P)$ that vary linearly from 0 at $P=P_{i-1}$ to 1 at $P=P_{i}$, linearly back to 0 at $P=P_{i+1}$, and 0 elsewhere (taking $P_{0}=P_{N}$ and $P_{N+1}=P_{1}$ ). Then, since $\Phi$ is a linear function of $y$ on each straight line segment,

$$
\oint \Phi \frac{\partial G}{\partial n} d s=\sum_{i=1}^{N} \Phi_{i} \int_{P_{i-1}}^{P_{i+1}} w_{i} \frac{\partial G}{\partial n} d s,
$$

where $\Phi_{i}=\Phi\left(P_{i}\right)$. Clearly also

$$
\oint \Phi_{Q} \frac{\partial G}{\partial n} d s=\Phi_{Q} \sum_{i=1}^{N} \int_{P_{i-1}}^{P_{i+1}} w_{i} \frac{\partial G}{\partial n} d s .
$$

Similarly, noting that $G=0$ on $\mathcal{S}$, and assuming $V$ to vary piecewise

\footnotetext{
${ }^{1}$ Note: $V$ is not the true unsteady fluid normal velocity on $\mathcal{I}$ because no account has been taken of the effect of the corresponding motion in the salt-water region on the pressure on $\mathcal{I}$. This simplification from the true unsteady problem avoids a $r \ln r$ type singularity at $\mathcal{I} \cap \mathcal{S}$, hence the time marching is more stable and the method more robust. It also significantly accelerates computations as there are fewer unknowns on $\mathcal{I}$.
} 
linearly over $\mathcal{I}$ and $\mathcal{F}$ between values of $V_{i}$ at points $P_{i}$, we have

$$
\int_{\mathcal{F} \cup \mathcal{I}} V G d s=\sum_{P_{i} \in \mathcal{F} \cup \mathcal{I}} V_{i} \int_{P_{i-1}}^{P_{i+1}} w_{i} G d s .
$$

Equation (8) can now be written in matrix form as

$$
\mathbf{A V}=-\mathbf{B} \Phi
$$

where

$$
\begin{aligned}
A_{j i} & =\int_{P_{i-1}}^{P_{i+1}} w_{i} G\left(P, P_{j}\right) d s_{P}, \\
B_{j i} & =\int_{P_{i-1}}^{P_{i+1}} w_{i} \frac{\partial G\left(P, P_{j}\right)}{\partial n_{P}} d s_{P}, \quad i \neq j, \\
B_{j j} & =-\sum_{\substack{i=1 \\
i \neq j}}^{N} B_{j i} .
\end{aligned}
$$

Here the index $j$ represents $Q$ in (4) $\left(Q=P_{j}\right)$; thus if $j$ takes values corresponding to each element of $\mathbf{V}$ we have sufficient equations to solve (12) to give

$$
\mathbf{V}=-\mathbf{A}^{-1}(\mathbf{B} \Phi)
$$

Note: $A_{j i}=0$ at points $Q \in(\mathcal{I} \cup \mathcal{F}) \cap \mathcal{S}$ and $P_{j} \in(\mathcal{I} \cup \mathcal{F}) \cap \mathcal{S}$, so these rows and columns must be excluded from $\mathbf{A}$ and rows from $\mathbf{B}$, and $V_{i}$ may be obtained at all points on $\mathcal{I} \cup \mathcal{F}$ except the end points. However, $V_{i}$ at these end points may be obtained independently through local analytical solutions.

Finally, boundary coordinates are incremented as explained above by

$$
\begin{aligned}
\Delta\left(x_{i} \mathbf{i}+y_{i} \mathbf{j}\right) & =V_{i} \mathbf{n}_{i} \Delta t \quad \text { on } \mathcal{I}, \\
\Delta\left(x_{i} \mathbf{i}+y_{i} \mathbf{j}\right) & =\left(V_{i}-V_{0} \mathbf{j} \cdot \mathbf{n}_{i}\right) \mathbf{n}_{i} \Delta t \quad \text { on } \mathcal{F} .
\end{aligned}
$$

However, points may also be moved a small but arbitrary distance tangentially along the boundary, and this is done to maintain a good distribution 
of points. For example, the interface end points must be moved tangentially as the boundary moves, unless $\mathcal{I}$ is perpendicular to $\mathcal{S}$, and other points on $\mathcal{I}$ are moved a proportion of this distance corresponding to the original distribution.

\section{Inclusion of a pumping well within the lens}

Let $W$ be the location of the pumping well, sink of strength $q<0$, near which $\Phi \rightarrow-q \ln r / 2 \pi$ as distance $r \rightarrow 0$ from $W$. Green's second identity requires $\nabla^{2} \Phi=0$ in $\Omega$, hence we exclude $W$ by adding to $\Gamma$ an additional circular contour $\mathcal{W}$ around $W$ of radius $\rho \rightarrow 0$ such that now $\Gamma=\mathcal{F} \cup \mathcal{S} \cup \mathcal{I} \cup \mathcal{W}$. On $\mathcal{W}$ the new boundary conditions are then (noting $\mathbf{n}$ points out of $\Omega$, that is, towards $W$ hence $\mathbf{r} \cdot \mathbf{n}=-1$ )

$$
\begin{aligned}
\Phi & =-\frac{q}{2 \pi} \ln \rho, \\
-\nabla \Phi \cdot \mathbf{n} & =-\frac{q}{2 \pi \rho} .
\end{aligned}
$$

$G$ is nonsingular at $W$, hence $G, \nabla G, \Phi$ and $\partial \Phi / \partial n$ are constant on $\mathcal{W}$ as $\rho \rightarrow 0$ and

$$
\lim _{\rho \rightarrow 0} \oint_{\mathcal{W}}\left(\left(\Phi-\Phi_{Q}\right) \frac{\partial G}{\partial n}-G \frac{\partial \Phi}{\partial n}\right) d s=-G(W, Q) q .
$$

This term is simply subtracted from the right side of (8), and equivalent equations, thus (16) becomes

$$
\mathbf{V}=-\mathbf{A}^{-1}(\mathbf{B} \boldsymbol{\Phi}-q G(W, \mathbf{P})) .
$$




\section{Complex representation}

The integrals in (13) and (14) are evaluated analytically. To do this, define the complex Green's function $\tilde{G}\left(z, z_{s}\right)$ whose real part is the real Green's function $G(P, Q)$ in which $z=x+\mathrm{i}(y-h)$ corresponds to the point $P \equiv(x, y)$ and $z_{s}=x_{s}+\mathrm{i}\left(y_{s}-h\right) \equiv Q \equiv\left(x_{s}, y_{s}\right)$, where $h=1 / \tan \alpha$; that is, for later convenience the origin for $z$ is at distance $h$ above sea-level where the two extrapolated straight lines $\mathcal{S}$ intersect in Figure 1. Representing the points $P_{i-1}, P_{i}$ and $P_{i+1}$ by $z_{L}, z_{C}$ and $z_{R}$ respectively, then

$$
w_{i}= \begin{cases}\left(z-z_{L}\right) /\left(z_{C}-z_{L}\right), & \text { between } z_{L} \text { and } z_{C}, \\ \left(z_{R}-z\right) /\left(z_{R}-z_{C}\right), & \text { between } z_{C} \text { and } z_{R},\end{cases}
$$

and the following equivalences hold:

$$
\begin{aligned}
\int_{z_{L}}^{z_{C}} \frac{\partial G}{\partial n}\left(\frac{z-z_{L}}{z_{C}-z_{L}}\right) d s & =\Im\left\{\tilde{G}_{C}-\frac{\int_{z_{L}}^{z_{C}} \tilde{G} d z}{z_{C}-z_{L}}\right\} \\
\int_{z_{C}}^{z_{R}} \frac{\partial G}{\partial n}\left(\frac{z_{R}-z}{z_{R}-z_{C}}\right) d s & =\Im\left\{-\tilde{G}_{C}+\frac{\int_{z_{C}}^{z_{R}} \tilde{G} d z}{z_{R}-z_{C}}\right\}, \\
\int_{z_{L}}^{z_{C}} G\left(\frac{z-z_{L}}{z_{C}-z_{L}}\right) d s & =\left|z_{C}-z_{L}\right| \Re\left\{\frac{\int_{z_{L}}^{z_{C}} \tilde{G} z d z-z_{L} \int_{z_{L}}^{z_{C}} \tilde{G} d z}{\left(z_{C}-z_{L}\right)^{2}}\right\}, \\
\int_{z_{C}}^{z_{R}} G\left(\frac{z_{R}-z}{z_{R}-z_{C}}\right) d s & =\left|z_{R}-z_{C}\right| \Re\left\{\frac{-\int_{z_{C}}^{z_{R}} \tilde{G} z d z+z_{R} \int_{z_{C}}^{z_{R}} \tilde{G} d z}{\left(z_{R}-z_{C}\right)^{2}}\right\}
\end{aligned}
$$

Noting the equivalences $\nabla G \equiv \operatorname{conj}(\partial \tilde{G} / \partial z)$ and $\mathbf{n} d s \equiv-\mathrm{i} d z$ the first two make use of the result

$$
\frac{\partial G}{\partial n} d s=\nabla G \cdot \mathbf{n} d s=\Re\left\{\frac{\partial \tilde{G}}{\partial z}(-\mathrm{i} d z)\right\}=\Im\left\{\frac{\partial \tilde{G}}{\partial z} d z\right\},
$$

and integration by parts, whereas the latter two use the result

$$
d s=\frac{|\Delta z|}{\Delta z} d z
$$


along a straight line between two points separated by $\Delta z$.

Finally, combining (13)-(15) and (23)-(27),

$$
\begin{aligned}
\mathbf{A}_{i j}= & \left|z_{C}-z_{L}\right| \Re\left\{\frac{\int_{z_{L}}^{z_{C}} \tilde{G} z d z-z_{L} \int_{z_{L}}^{z_{C}} \tilde{G} d z}{\left(z_{C}-z_{L}\right)^{2}}\right\} \\
& +\left|z_{R}-z_{C}\right| \Re\left\{\frac{-\int_{z_{C}}^{z_{R}} \tilde{G} z d z+z_{R} \int_{z_{C}}^{z_{R}} \tilde{G} d z}{\left(z_{R}-z_{C}\right)^{2}}\right\}, \\
\mathbf{B}_{i j}= & \Im\left\{\frac{\int_{z_{C}}^{z_{R}} \tilde{G} d z}{z_{R}-z_{C}}-\frac{\int_{z_{L}}^{z_{C}} \tilde{G} d z}{z_{C}-z_{L}}\right\} .
\end{aligned}
$$

\section{Green's function and its integrals}

To complete the solution we seek an analytic complex function $\tilde{G}(z)$, which will automatically be harmonic in $\Omega$, whose real part satisfies (5). We also require integrals $\int \tilde{G} d z$ and $\int \tilde{G} z d z$ appearing in (30)-(31). As imaginary parts of $\tilde{G}$ are involved in these integrals we must also ensure that appropriate corrections are applied if the path of integration crosses a branch cut in $\tilde{G}$.

The Green's function for wedge angle $2 \alpha=a \pi(0<a<1)$, obtained by mapping the right half of the $\zeta$-plane in which $\Re\{\tilde{G}\}=0$ on the imaginary axis, achieved by placing a source and sink symmetrically about this axis, onto the island wedge in the $z$-plane is

$$
2 \pi \tilde{G}\left(z, z_{s}\right)= \begin{cases}\ln \left(1-u_{1}\right)-\ln \left(1+u_{2}\right)+\ln \left(\zeta_{s} / \bar{\zeta}_{s}\right), & \left|u_{1}\right| \leq 1 \\ \ln \left(1-1 / u_{1}\right)-\ln \left(1+1 / u_{2}\right) \pm \mathrm{i} \pi, & \left|u_{1}\right| \geq 1\end{cases}
$$

where $u_{1}=\zeta / \zeta_{s}, u_{2}=\zeta / \bar{\zeta}_{s}, \zeta=(\mathrm{i} z)^{1 / a}, \zeta_{s}=\left(\mathrm{i} z_{s}\right)^{1 / a}$ and the branch cut has been arranged to lie on the line $z / z_{s}>1$ and real. 
The integrals of $\tilde{G}$ can be shown, by expanding the log terms, to be

$$
\begin{aligned}
2 \pi \int_{z_{1}}^{z_{2}} \tilde{G} d z & =I_{0}\left(z_{2}, z_{s}, a\right)-I_{0}\left(z_{1}, z_{s}, a\right)+\delta 2 \pi \mathrm{i}\left(z_{c}-z_{s}\right), \\
2 \pi \int_{z_{1}}^{z_{2}} \tilde{G} z d z & =I_{1}\left(z_{2}, z_{s}, a\right)-I_{1}\left(z_{1}, z_{s}, a\right)+\delta \pi \mathrm{i}\left(z_{c}^{2}-z_{s}^{2}\right),
\end{aligned}
$$

where for $\left|u_{1}\right| \leq 1$

$$
I_{0}\left(z, z_{s}, a\right)=-a z \sum_{k=1}^{\infty} \frac{u_{1}^{k}-\left(-u_{2}\right)^{k}}{k(a+k)}+\frac{z}{a}\left(\ln \left(\mathrm{i} z_{s}\right)-\ln \left(-\mathrm{i} \bar{z}_{s}\right)\right),
$$

and for $\left|u_{1}\right| \geq 1$

$$
I_{0}\left(z, z_{s}, a\right)=-a z \sum_{k=1}^{\infty} \frac{u_{1}^{-k}-\left(-u_{2}\right)^{-k}}{k(a-k)}+\pi\left(z_{s} \cot a \pi+\frac{\bar{z}_{s}}{\sin a \pi}\right) \mp \mathrm{i} \pi\left(z_{s}-z\right),
$$

and

$$
I_{1}\left(z, z_{s}, a\right)=I_{0}\left(\frac{z^{2}}{2}, \frac{z_{s}^{2}}{2}, 2 a\right) .
$$

Branch cut crossings are corrected using $\delta=+1$ if the integration path crosses the branch cut in the anti-clockwise direction, -1 for a clockwise crossing, or 0 if the branch cut is not crossed. $z_{c}$ is the point of intersection of the integration path with the branch cut.

\section{Some results}

After normalisation, the parameters of this problem reduce to the salt-fresh water density ratio $\gamma$, the rainfall relative to permeability $V_{0}$, and the side slope $\alpha=a \pi / 2$ (measured from the vertical). Results are presented below for $\gamma=1.025, \alpha=75^{\circ}$ and $V_{0}=0.01$. The latter is based on an annual effective 
rainfall, reduced for evaporation and runoff, of $10^{-8} \mathrm{~m} / \mathrm{s}(316 \mathrm{~mm} /$ year $)$ and soil permeability of $10^{-6} \mathrm{~m} / \mathrm{s}$, which is regarded as low [3]. The corresponding time scale based on a length of $10 \mathrm{~km}$ say is approximately 300 years, so it is legitimate to ignore seasonal variations in rainfall and tidal fluctuations of the sea level. Of these quantities, permeability is the most variable. However, higher permeabilities are of lesser interest as they give lower values of $V_{0}$, and the lens shape asymptotically approaches that given by a one dimensional solution based on the Dupuit-Forchheimer assumption that velocities are horizontal and constant throughout the depth $[1,4]$. Very low permeabilities $\left(<10^{-8} \mathrm{~m} / \mathrm{s}\right)$ are also of limited interest as then $V_{0}>1$, implying more rainfall than the ground is capable of absorbing.

Figure 2 illustrates sensitivity to the number of elements. Element density has been increased nearer $\mathcal{F} \cap \mathcal{S}$ and $\mathcal{I} \cap \mathcal{S}$, where greater rates of change of the shape of $\Gamma$ are expected. It was found that the steady state solution was independent of the assumed starting location of $\Gamma$, unless $\Gamma$ initially approached the sink $W$ too closely for a solution to be obtained. Acceptable results are obtained with 20 elements on each of $\mathcal{I}$ and $\mathcal{F}$, while negligible visible improvement is obtained with more than 40 elements. However, smaller elements require correspondingly smaller time steps to maintain stability, hence correspondingly more time steps to achieve convergence. A cosine element distribution was used between critical points, in which the interval $[0,1]$ is divided into $n$ elements defined by end points $x_{i}=\frac{1}{2}(1-\cos (i \pi / n))$ for $i=0,1,2, \ldots, n$, giving locally quadratic refinement at both ends. Allowing for the corresponding additional time steps required the total computation times increase with the fourth power of element number.

The lower half of Figure 2 shows the lens with the well $W$ at its optimum location (that is, that which maximises the well pumping rate $q$ without entraining salt water) and at half its maximum pumping rate. As $q$ is increased to approach its maximum value the results do not qualitatively differ. The requirement of a stagnation point on $\mathcal{I}$ at steady state ensures a finite separation of $\mathcal{I}$ from $W$. However, once the maximum $q$ is reached the steady 

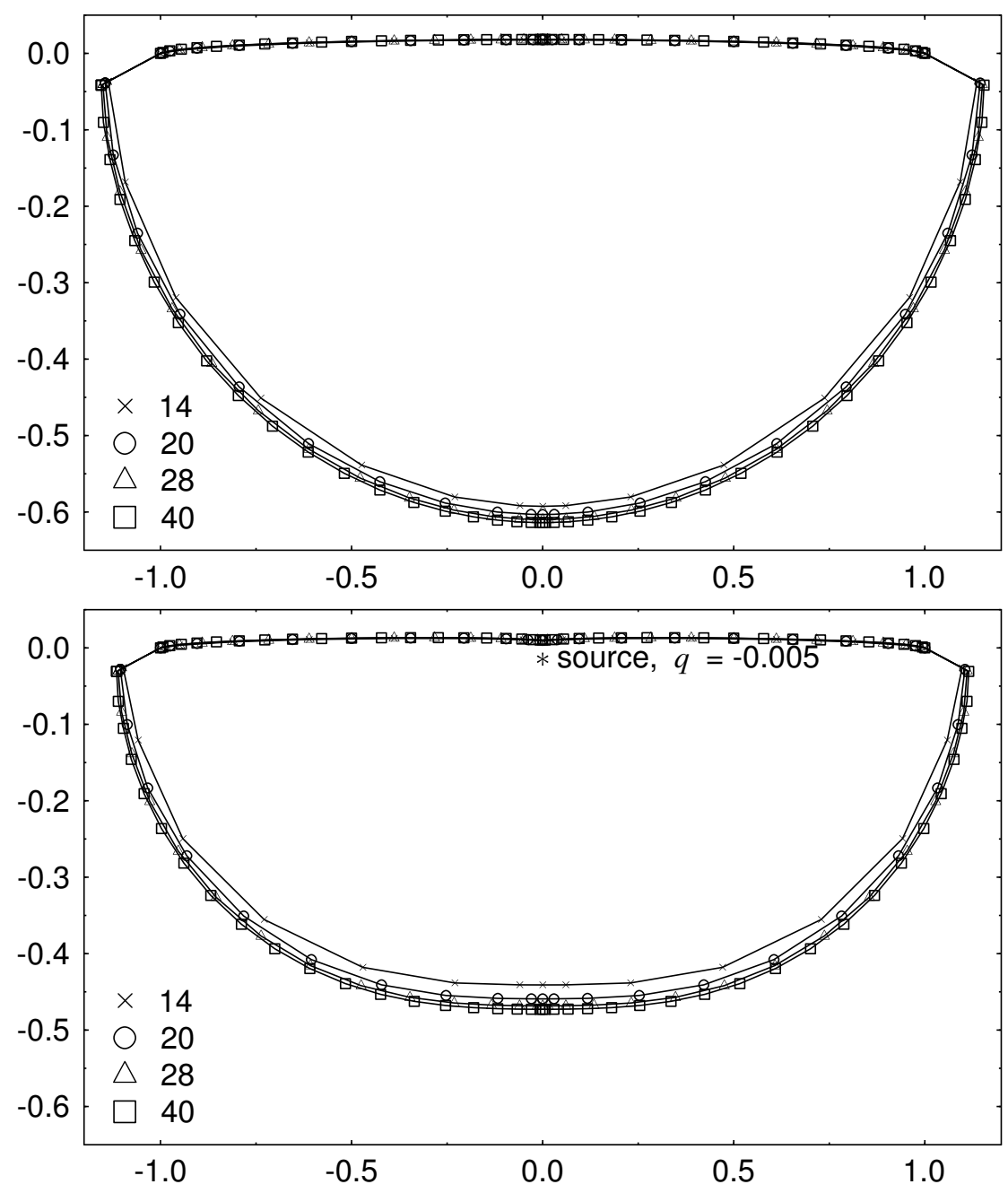

FIGURE 2: Showing convergence as number of elements is increased from 14 to 40 on each of $\mathcal{I}$ and $\mathcal{F}$ : upper figure without source; lower figure with source at $(0,-0.015)$ of strength $q=-0.005$. Vertical exaggeration is $2 \times$. Results are shown for $\gamma=1.025, \alpha=75^{\circ}$ and $V_{0}=0.01$. 
state solution abruptly ceases to exist. This abrupt transition renders it impossible to estimate critical value of $q$ by extrapolation, and it must be found by gradually ramping up $q$ until the solution fails. Near the critical $q$ convergence or divergence may be very slow, and it may require a long simulation to decide conclusively whether or not $\mathcal{I}$ or $\mathcal{F}$ will be entrained by $W$. Thus the present computations have adopted 20 elements on each of $\mathcal{F}$ and $\mathcal{I}$.

Figure 3 shows the maximum well pump rate as a function of vertical position. The optimum position is at approximately $y=-0.015$, slightly below sea level, where the inflow $\left(V_{0}=0.01\right.$ over a width of 2$)$ is evenly divided between outflow at $\mathcal{S}$ and extraction at the well. Above this point $\mathcal{F}$ becomes entrained as $q$ exceeds its limiting value, while $\mathcal{I}$ becomes entrained for a well below this optimum location. Clearly $\mathcal{I}$ is much more sensitive to disturbance than $\mathcal{F}$ because of the smaller density difference, hence the optimum well location is much closer to $\mathcal{F}$ then $\mathcal{I}$.

\section{Conclusions}

This efficient technique for solving the groundwater lens problem shows that acceptable results may be obtained with as few as 20 elements on each of the free surface and interface. The method is structured so that is may easily be extended to the true unsteady case, although this would require numerical problems due to a singularity at $\mathcal{I} \cap \mathcal{S}$ to be overcome.

One-dimensional solutions are known for the lens problem, but a twodimensional solution, such as presented above, is necessary to investigate the effect of well height. The optimum location was found to be just below sea level, nearer the upper surface, but this optimum is less sensitive to erring on the lower side than the higher, so it is recommended that a well be placed at or slightly below the optimum location. Further work is being conducted, with this method, to determine optimum sink locations for a wide variety of well locations in groundwater lenses. 

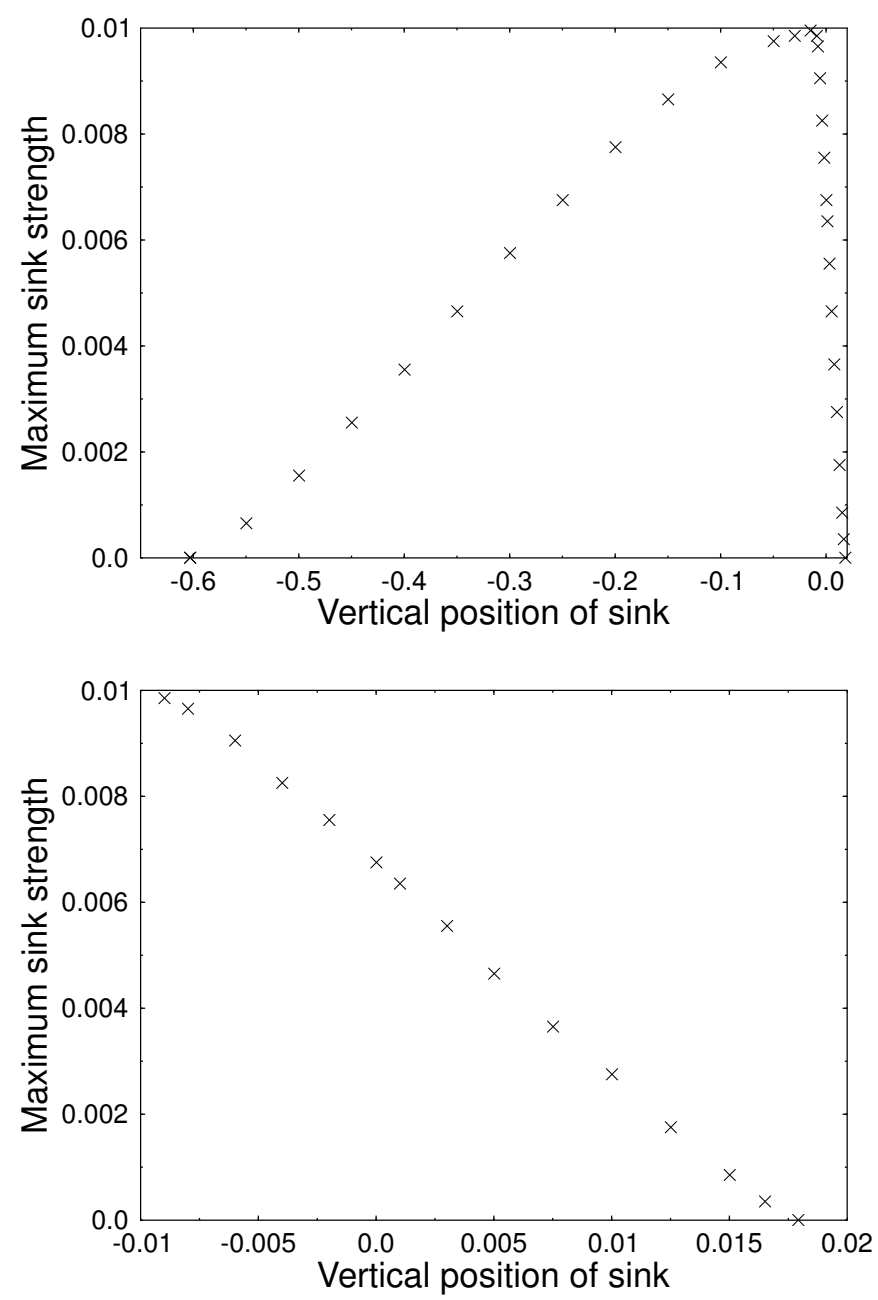

FiguRe 3: Maximum pumping rate $(-q)$ as function of well height (lower figure shows detail for well located near $\mathcal{F}$ ). Results are shown for $\gamma=1.025$, $\alpha=75^{\circ}$ and $V_{0}=0.01$. 
Acknowledgements: This work was funded by Australian Research Council grant number DP0450225 administered through the School of Mathematics and Physics, University of Tasmania.

\section{References}

[1] Herman Bouwer. Groundwater Hydrology. McGraw-Hill, New York, 1978. C457

[2] G. C. Hocking and L. K. Forbes. The lens of freshwater in a tropical island -2d withdrawal. Computers and Fluids, 33:19-30, 2004. doi:10.1016/S0045-7930(03)00035-5 C449

[3] T. William Lambe and Robert V. Whitman. Soil Mechanics. Wiley, 1969. C457

[4] A. J. Raudkivi and R. A. Callander. Analysis of Groundwater Flow. Edward Arnold, 1976. C457 